\title{
Sexual Education and Women Empowerment in Health: A Review of the Literature
}

\author{
Khadigeh Mirzaii Najmabadi $^{\mathbb{D}}$, Farangis Sharifi ${ }^{2^{*}}$
}

\begin{abstract}
Objectives: Women constitute almost $50 \%$ of the world population and play a number of roles in the community. The present study aimed to assess the relationship between sex education and women empowerment in health.

Methods: Based on the aim of the study, the data were obtained reviewing the related literature on electronic and non-electronic websites and using equivalent keywords published until the last week of February 2018. In addition, mesh terms and key words were included in this research. Inclusion criteria were articles published from 2005 to February 2018. All the observation or interventional studies including ten articles which met the search criteria were studied.

Results: The results revealed that sex education has different effects on sexual attitudes or activities of women. Further, it was effective in increasing the sexual autonomy of female college students. Furthermore, health providers' contribution may improve sexual autonomy. In the current study, sex education programs were effective at increasing HIV/AIDS ( Human Immunodeficiency Virus/Acquired Immune Deficiency Syndrome) knowledge and condom use, reducing the risk of unprotected intercourse and sexually transmitted infections (STIs), as well as unplanned pregnancy and abortion. Finally, abstinence-plus sex education programs increased health knowledge while they reduced risky sexual behaviors.

Conclusions: In general, sex education is a critical method of women empowerment in health through increasing their health knowledge and related behaviors. Therefore, a compilation program of sex education is more useful. Accordingly, seeking to place sex training in the educational curriculum in accordance with the culture of the countries is a necessity.

Keywords: Empowerment, Health, Sex education
\end{abstract}

\section{Introduction}

Women constitute nearly $50 \%$ of the world population (1) while gender inequality restricts their growth (2). In other words, women are worshipped as a goddess while they are simultaneously oppressed, suppressed, depresses, exploited, and victimized by a male-dominated society. This social history is concealed behind the myth that women are naturally inferior to men $(3,4)$.

Women occupy many status positions at a given point of time and play different roles in the kinship system, family system, and wider social system. In addition, their position in society is determined by the integration of different situations (5).

Empowerment in a broader sense is defined as the expansion of people's ability to make strategic choices in a context which could have a wide range of meanings and can vary from one society to another and even from one individual to another. Further, some define empowerment as a process of awareness which leads to greater participation, effective decision-making power, control, and thus transformative action. Furthermore, the economic, social, political, religious, cultural, and psychological factors affect empowerment $(1,2,6,7)$.

An essential concept in empowerment is power; autonomy and economic components are considered other elements of empowerment. Moreover, Freire believed that education as political action is a significant part of the empowerment process. According to him, the disadvantaged individuals can become empowered by learning about social inequality $(6,8,9)$. Additionally, empowerment is related to have control over material assets, intellectual resources, and ideology (7).

Theorists disagree about how to define the concept of empowerment and this extends to discussions of the girls' sexual empowerment (10). One of the eight goals, that is, millennium development goals (MDGs), is focused on improving maternal health and is lauded as the key to achieve several other MDGs. MDGs are measured by six indicators including three indicators particularly related to comprehensive sex education, namely, achieving a reduction in teenage pregnancy rates, an increase in contraceptive rates, and meeting the unmet needs for family planning $(11,12)$.

In addition, sex education should include social and moral behavior more than biological specifics. This may encompass various factors such as delayed initiation of sexual activity, reduction in unplanned and early pregnancies and their associated complications, fewer

Received 5 June 2018, Accepted 27 September 2018, Available online 19 October 2018

${ }^{1}$ Department of Midwifery, School of Nursing and Midwifery, Mashhad University of Medical Sciences, Mashhad, Iran. ${ }^{2}$ Student Research Committee, School of Nursing and Midwifery, Mashhad University of Medical Sciences, Mashhad, Iran

*Corresponding Author: Farangis Sharifi, Tel: (+98) 51 38591511, Fax: +98-5138598016, Email: sharifif961@mums.ac.ir 
unwanted children, reduced risks of sexual abuse, greater completion of education and later marriages, reduced recourse to abortion and the consequences of unsafe abortion, and curb the spread of sexually transmitted diseases including HIV. Sex is a very sensitive subject and public discussion on sexual matters are considered a taboo in some of the societies. Further, sex education has constantly attracted objections and apprehensions from many quarters $(13,14)$. Some have high expectations of sexuality education and consider it as a solution to a series of complex, persistent, and multifaceted social issues including unwanted pregnancies, sexually transmitted infections (STI), human immunodeficiency virus (HIV), and sexual abuse. However, others believe that sexuality education by itself fails to prevent any negative consequences of sexuality. According to the World Health Organization (WHO), sex education programs are considered as a need and contain different types and functions. For example, comprehensive sex education programs can prevent sexual dysfunction, create safe sexual behavior and mental health, increase positive health behaviors and sexual identity, and establish effective family health $(15,16)$. Furthermore, sex relationship education has three main elements. The first component is within a clearly defined moral framework, namely, that of respect, marriage, and family life. The second element contains social skills and the third and final element includes children and young people who should be supported so that they make the 'informed decision' in order to delay sexual activity and avoid unplanned pregnancy (12).

The main argument regarding the content of sex education is whether to teach abstinence-only curricula or curricula which include abstinence, as well as information about birth control and sexual health resources (17). Moreover, adolescent sexual identity develops within the context of gender-role stereotypes and youth sexual scripts are received from individuals and institutions within their culture (18). In many societies, attitudes and laws discourage public discussion of sexuality and sexual behavior, and social norms may perpetuate harmful conditions, for example, gender inequality in relation to sexual relationships, family planning, and modern contraceptive use (19).

In a study conducted in Iran, the average amount of attention paid by families, as well as the average amount of attention to sexual awareness, ethics, and future in the sexual education of children were lower compared to the average level (20). In another study, the main barriers for accessing the identified sexual and reproductive health information were classified into social and cultural barriers (i.e., taboos), structural and administrative barriers (i.e., inappropriate structure of the health system), political barriers (i.e., lack of an adopted strategy by the government), and no use of religious potential (21).

Given the importance of empowering women in line with the MDGs and its relationship with sexual education and considering the need for sexual education, this women empowerment should be part of the educational system $(11,20,22)$. Accordingly, given the role of sex education in the sexual and reproductive behaviors in some countries (23) and considering the lack of any review study in this area in recent years, the current study was conducted aiming at reviewing the appropriate evidence in order to evaluate the association between sex education and women empowerment in health. In other words, the study sought to identify the effect of sex education on knowledge or attitude of women. Additionally, it was attempted to determine the effect of sex education on their behavior and finally, to compare the role of sex education and abstinence as investigated in the related literature.

\section{Methods}

Sample Selection and Characteristics

Considering the aims of the study, the related data were obtained from the literature review using the PubMed, Scopus, Web of Science, Science Direct, and Psych-net, as well as Persian databases including Iran Medex, SID, and Magiran and employing equivalent keywords up to the last week of February 2018. In addition, mesh terms and key words included "teaching, training, education, sexual, sex, power, empowerment, knowledge, attitude, and behavior". Further, the search was expanded to the title, abstract, or keywords, along with using each combination of "AND" and "OR" between the search terms. Furthermore, the references of the articles selected for electronic reviews and other related reviews were manually checked to find the related articles for the current study. Finally, in the process of extraction, one of the investigators reviewed both the titles and abstracts of the articles in order to determine their suitability for inclusion.

Inclusion criteria for the study sample were articles published from 2005 to (February) 2018. After an independent assessment by the authors, those studies which included sex education in women were considered to be investigated due to their consequences. Moreover, all the observation or interventional studies meeting the search criteria were included in the current study including a total of 10 articles (5 experimental and 5 descriptive studies), the characteristics of which are provided in online Supplementary file 1.

\section{Results}

Sex education was found to have many goals, some of which are common in different studies (24). Sex education is a topic of interest by many people and thus it's important whether appropriate courses have a significant impact on increasing the women's knowledge of their empowerment.

In a descriptive comparative study in Korea, sex education at different time points during the school-aged years was reported to have differential influences on sexual attitudes. However, sex education during the college years had no effect on sexual attitudes or activities of both male 
and female students (25).

Additionally, in a survey of a sexual education program in the context of a school-community collaboration, the links between several components of sexual empowerment including gender ideology, sexual knowledge, and contraceptive beliefs were assessed. Structural equation models suggested that traditional attitudes toward women were significantly related to hegemonic masculinity ideology among both boys and girls, which was in turn negatively associated with safer contraceptive beliefs (18).

In addition, the results of an assessment regarding the effects of the SOMOS sex education program on the attitudes towards sexuality, in general, after interventional education, confirmed that the program fostered the development of positive attitudes towards sexuality while it significantly decreased negative attitudes towards masturbation. It was further found that the sexual experience of the students progressively increased regardless of the group they belonged to (26).

Furthermore, the sexual education program was reported to be effective in increasing female college students' sexual autonomy in another study. Nursing professors majoring at women's health nursing may contribute to improving the college students' sexual autonomy by opening the sexual education program as a general elective curriculum. Moreover, strategies to improve sexual knowledge need to be considered for better effectiveness of such programs (27).

It is noteworthy that in some countries, sexual education was found to affect reproductive health and STI/HIV behavioral risks, along with influencing the knowledge. Several interventional studies discussed in this section demonstrated efficacy in their study populations.

Due to a significant increase in the rate of HIV/AIDS in Dominican Republic a Encuesta Nacional de Jovenes, (ENJOVEN), a collaborative survey (between the Instituto de Estudios de Poblaciöny Desarrollo, Asociaciön Dominicana Pro Bienestar de la Familia, Reproductive Health, Centers for Disease Control and Prevention) was performed on this country and the results indicated that sex education programs were effective in increasing HIV/ AIDS knowledge and condom use in young people (28).

Additionally, school-based sexual education was reported to reduce the risk of unprotected intercourse and STIs in early adulthood among British university students. Based on the findings, being influenced by the friends in adolescence was believed to have a positive effect on the risk of STIs in later life and age of first intercourse associated with unprotected intercourse (29).

In a study across North America schools, women received a combination of contraceptive and abstinence education and those receiving primarily abstinence education were least likely to experience an unplanned pregnancy. In addition, religious identification and the type of sex education received from the parents were significantly related to unplanned pregnancy. Finally, those receiving primarily abstinence education in school were least likely to experience unplanned pregnancy (30).

In northern Taiwan, randomized controlled trial on sexual health knowledge, attitudes, and sexual self-efficacy were significantly greater for women in the "theory-based postpartum program related to sexual health education" group. However, contraceptive self-efficacy was not significantly different in the two groups (31).

Further, the results of a study performed never-married heterosexual adolescents revealed that teaching about contraception was not associated with increased risk of adolescent sexual activity or STD. Adolescents who received comprehensive sex education had a lower risk of pregnancy compared to adolescents who received abstinence-only or no sex education. Furthermore, based on the findings, abstinence-only education failed to reduce the likelihood of engaging in vaginal intercourse while comprehensive sex education was marginally associated with a lower likelihood of reporting having engaged in vaginal intercourse. Neither abstinence-only nor comprehensive sex education significantly decreased the likelihood of reported STD diagnoses (23).

Moreover, a randomized stratified quasi-experimental study was conducted on Mazandaran Medical University students of Iran and the results demonstrated that sex education had an effect on appropriate sexual behavior, mental health, sexual problem prevention, family health, and appropriate gender identity. Additionally, its effect varied between male and female students (32).

\section{Discussion}

This research reviewed appropriate evidence in order to investigate the relationship between sex education and women empowerment in health. To this end, empowerment was considered in several health areas.

\section{Knowledge or Attitude of Women}

Most of the results reported the effect of sex education on increasing the women's knowledge. In addition, the results of some studies indicated that more progressive attitudes toward girls and women, less agreement with hegemonic masculinity ideology, and increases in sexual health, resource knowledge, and positive attitudes of the women $(18,26,28,33)$ were effective in this respect. Adamczyk and Greif in their study found that gender empowerment was partially related to the relationship between education and HIV/AIDS knowledge (34). It should be noted that in some studies investigated in the current study, sex education had no effect on individuals' attitudes. The results of these studies suggested extending the role of nurses, midwives, and professional health educators in sex education programs $(25,27)$. Further, Kim and Free indicated that despite the promising results in some trials, the overall findings failed to provide convincing evidence respecting the role of peer-led education in improving the sexual outcomes among the adolescents (35). Furthermore, Choi 
and $\mathrm{Ha}$ recommended that an integrated sex education program was appropriate for college students (36). Eventually, Agbemenu et al. decorated that nurses can serve as liaisons between the adolescents, community, and government in promoting sex education programs (11).

Health Behaviors of Women

Attitudes about gendered inequality mediate the relationship between women's education and safer-sexrelated behaviors. In the current study, sex education programs were confirmed to be effective in increasing HIV/AIDS knowledge and condom use, reducing the risk of unprotected intercourse and STIs, unplanned pregnancy and abortion, appropriate behavior, and mental health, and finally, preventing the sexual problems, family health, and appropriate gender identity (28, 30, 32). Moreover, the results of several studies represented that community empowerment-based HIV prevention was associated with significant improvements across HIV outcomes and setting $(33,37,38)$. Additionally, in other reviews, the programs which addressed gender or power were five times as likely to be effective as those which did not focus on this issue. Totally, $80 \%$ of such programs were associated with a significantly lower rate of STIs or unintended pregnancies $(23,39)$. However, in the study by DiCenso et al, primary prevention strategies evaluated to date are unable to delay the initiation of sexual intercourse, improve the use of birth control among young men and women, or reduce the number of pregnancies in young women (40). In addition, Kim and Free found that peerled education review failed to improve sexual outcomes among adolescents (35).

Sex education, along with abstinence is effective in sexual health behavior while abstinence alone has no impact on this category (23). Carr and Packham in their study observed that state-level mandates of abstinence education had no effect on teen birth rates or abortion rates whereas state-level policies found to affect the rate of teen sexually transmitted disease in some states (41). Comprehensive sex education or abstinence-plus programs were reported to increase STI knowledge while reducing the risk-taking behaviors in adolescents and young adults (42).

Further, an important finding of a study implemented in Iranian indicated that sex education effect was different between male and female students. This problem was due to the specified culture of this area (30).

In the current study, sex education was provided from different sources (28-30) whereas some women were more satisfied with sex education from informal sources compared to those obtained from the parents (29). Furthermore, comprehensive sexuality education was most effective in promoting sexual health in the present study, which is in line with the findings of Braeken and Cardinal (15).

Programs designed to promote skills and those which are modified based on the effectiveness evaluations are most likely to have an impact on the rate of pregnancies and STIs among young people. However, such programs may not be used in some schools due to their policy and cultural constraint; therefore, this leads to the lack of optimally effective health promotion. Accordingly, health promoters by targeting communities and legislators should affirm these challenges and facilitate the adoption and implementation of effective programs regarding sexual health promotion and women empowerment.

\section{Limitations of the Study}

In fact, considering that women's empowerment and sexual education programs are under the influence of cultural constraints of several countries, the study was not performed in all areas and access to data in this area was difficult. Moreover, some investigated papers were conducted retrospectively and thus these types of articles have their own disadvantages.

\section{Conclusions}

In general, sex education is a critical means for women empowerment in health through increasing women's knowledge and related behaviors. Sex education for individuals, especially young people can be performed by school-based educations, parents' educations and peer educations. Additionally, midwife, nurse, and health providers' educations should be considered in the programs and totally, a compilation program is more useful in this respect. Lifestyle is a dynamic chain in all aspects of human life and plays an important role in the health of humans (43). The most important aspect regarding sexual education is to use preservatives and contraceptive methods, as well as rescue the individual as a result of his/ her own actions. This favors the development of a sense of citizenship, respect, commitment, self-care, and care for others (44). Although implementing sex education programs is influenced by the culture of the countries and regions, it is necessary to attempt to place these training on the educational curriculums in accordance with the culture of the countries and that sexual reproductive health should be firmly established as a priority for government leaders and policymakers $(21,45)$. Eventually, based on the findings, abstinence-plus sex education programs were reported to increase health knowledge and reduce risky behaviors.

\section{Conflict of Interests}

Authors declare that they have no conflict of interests.

\section{Ethical Issues}

Not applicable.

\section{Financial Support}

None declared. 


\section{Acknowledgments}

Our special thanks go to professors and Vice-chancellor for Research and Student Research Committee of Mashhad University of Medical Sciences and authors of the articles which were reviewed in the present study.

\section{Supplemenatry Data}

Supplemenatry file 1 contains the Characteristics of 10 Studies Included in the Present Study.

\section{References}

1. Kermode M, Herrman H, Arole R, White J, Premkumar R, Patel V. Empowerment of women and mental health promotion: a qualitative study in rural Maharashtra, India. BMC Public Health. 2007;7:225. doi:10.1186/1471-2458-7225

2. Ibanez M, Khan S, Minasyan A, Sahoo S, Balasubramanian P. The impacts of interventions for female economic empowerment at the community level on human development: a systematic review of the evidence in low-and middle-income countries. The Campbell Collaboration; 2017.

3. Singhal V. Empowerment of Women (Review of Literature). Laxmi Nagar: Sunrise Publications; 2016.

4. Chhabra S. Legislative and Judicial Perspective of Women Empowerment. 2015.

5. Kumar V, Mohanty S, Kumar A, et al. Effect of communitybased behaviour change management on neonatal mortality in Shivgarh, Uttar Pradesh, India: a cluster-randomised controlled trial. Lancet. 2008;372(9644):1151-1162. doi:10.1016/s0140-6736(08)61483-x

6. Fereidouni S, Mehran G, Mansourian Y. Female Empowerment in Iran: The Voice of Iranian University Students. Higher Education Quarterly. 2015;69(4):366-385. doi:10.1111/hequ.12079

7. Ravichandran K, Gomala VR. Women empowerment in India-milestones and challenges. International Journal of Social Science and Interdisciplinary Research. 2014;3(7):230-238.

8. Freire P. Education for critical consciousness. Bloomsbury Publishing; 1973.

9. Freire P. Pedagogy of the oppressed. New York: Herder and Herder; 1970.

10. Peterson ZD. What is sexual empowerment? A multidimensional and process-oriented approach to adolescent girls' sexual empowerment. Sex Roles. 2010;62(5-6):307-313. doi:10.1007/s11199-009-9725-2

11. Agbemenu K, Schlenk EA. An integrative review of comprehensive sex education for adolescent girls in Kenya. J Nurs Scholarsh. 2011;43(1):54-63. doi:10.1111/j.15475069.2010.01382.x

12. SpencerG, MaxwellC, Aggleton P.What does 'empowerment' mean in school-based sex and relationships education? Sex Educ. 2008;8(3):345-356. doi:10.1080/14681810802218437

13. Tripathi N, Sekher TV. Youth in India ready for sex education? Emerging evidence from national surveys. PLoS One. 2013;8(8):e71584. doi:10.1371/journal.pone.0071584

14. Mturi AJ, Hennink MM. Perceptions of sex education for young people in Lesotho. Cult Health Sex. 2005;7(2):129143. doi:10.1080/13691050412331321285

15. Braeken D, Cardinal M. Comprehensive sexuality education as a means of promoting sexual health. Int J Sex Health. 2008;20(1-2):50-62. doi:10.1080/19317610802157051

16. Behboodi Moghadam Z, Rezaei E, Khaleghi Yalegonbadi F, et al. The effect of sexual health education program on women sexual function in Iran. J Res Health Sci. 2015;15(2):124-128.

17. Raitz KL. Youth empowerment in sex education. Boulder: University of Colorado; 2015.

18. Grose RG, Grabe S, Kohfeldt D. Sexual education, gender ideology, and youth sexual empowerment. J Sex Res. 2014;51(7):742-753. doi:10.1080/00224499.2013.809511

19. UNESCO. International technical guidance on sexuality education: An evidence-informed approach for schools, teachers and health educators. Volume I and II. 2009. https://www.unfpa.org/sites/default/files/pub-pdf/ITGSE. pdf.

20. Sobhaninejad M, Homaie R, Aliin H. Investigation of the Sexual Education Style of Tehran Families Counseling Research (Updates and Consultation Research). 2007;6(21):27-48.

21. Shariati M, Babazadeh R, Mousavi SA, Najmabadi KM. Iranian adolescent girls' barriers in accessing sexual and reproductive health information and services: a qualitative study. J Fam Plann Reprod Health Care. 2014;40(4):270275. doi:10.1136/jfprhc-2013-100856

22. Kelly A. Consent in Sex Education: Teacher Perspectives on Teaching Consent in the Updated Health and Physical Education Curriculum. University of Toronto; 2017.

23. Kohler PK, Manhart LE, Lafferty WE. Abstinence-only and comprehensive sex education and the initiation of sexual activity and teen pregnancy. J Adolesc Health. 2008;42(4):344-351. doi:10.1016/j.jadohealth.2007.08.026

24. Reid D. School sex education and the causes of unintended teenage pregnancies: -a review. Health Educ J. 1982;41(1):411. doi:10.1177/001789698204100103

25. Shin KR, Park H, Cha C. Sex education during the schoolaged years influences sexual attitudes and sexual health in college: a comparative study from Korea. Nurs Health Sci. 2011;13(3):328-334. doi:10.1111/j.1442-2018.2011.00622.x

26. Sevilla DH, Lara-Ortega F, Fernandez Hawrylak M. Evaluation of the Effects of the Sexual Education Programme SOMOS on Sexual Experience and Attitudes of Adolescents Towards Sexuality. [Evaluación de los efectos del Programa de Educación Sexual SOMOS sobre la experiencia sexual y las actitudes hacia la sexualidad de adolescents]. Revista de Psicodidáctica. 2016;21(2):321337. doi:10.1387/RevPsicodidact. 14300

27. Chun N. Effect of sexual education program on female college student's sexual knowledge and sexual autonomy. Korean J Women Health Nurs. 2012;18(2):108-116. doi:10.4069/kjwhn.2012.18.2.108

28. Minaya J, Owen-Smith A, Herold J. The impact of sex education on HIV knowledge and condom use among adolescent females in the Dominican Republic. Int J Adolesc Med Health. 2008;20(3):275-282. doi:10.1515/ IJAMH.2008.20.3.275

29. Vivancos R, Abubakar I, Phillips-Howard P, Hunter PR. School-based sex education is associated with reduced risky sexual behaviour and sexually transmitted infections in young adults. Public Health. 2013;127(1):53-57. doi:10.1016/j.puhe.2012.09.016

30. Williams MT, Bonner L. Sex education attitudes and outcomes among North American women. Adolescence. 
2006;41(161):1-14.

31. Lee JT, Tsai JL, Tsou TS, Chen MC. Effectiveness of a theory-based postpartum sexual health education program on women's contraceptive use: a randomized controlled trial. Contraception. 2011;84(1):48-56. doi:10.1016/j. contraception.2010.11.008

32. Mahmoudi G, Hassanzadeh R, Heidari G. The effect of sex education on family health on Mazandran medical university students. The Horizon of Medical Sciences. 2007;13(2):64-70.

33. Dei Jnr LA. The efficacy of HIV and sex education interventions among youths in developing countries: a review. Public Health Research. 2016;6(1):1-17. doi:10.5923/j.phr.20160601.01

34. Adamczyk A, Greif M. Education and risky sex in Africa: Unraveling the link between women's education and reproductive health behaviors in Kenya. Soc Sci Res. 2011;40(2):654-666. doi:10.1016/j.ssresearch.2010.12.003

35. Kim CR, Free C. Recent evaluations of the peer-led approach in adolescent sexual health education: a systematic review. Perspect Sex Reprod Health. 2008;40(3):144-151. doi:10.1363/4014408

36. Choi MS, Ha NS. A study on knowledge, attitude, and experience in sex and sexual autonomy of college students. Korean J Women Health Nurs. 2004;10(4):318-330.

37. Kerrigan DL, Fonner VA, Stromdahl S, Kennedy CE. Community empowerment among female sex workers is an effective HIV prevention intervention: a systematic review of the peer-reviewed evidence from low- and middleincome countries. AIDS Behav. 2013;17(6):1926-1940. doi:10.1007/s10461-013-0458-4

38. Fonner VA, Armstrong KS, Kennedy CE, O'Reilly KR, Sweat MD. School based sex education and HIV prevention in low- and middle-income countries: a systematic review and meta-analysis. PLoS One. 2014;9(3):e89692. doi:10.1371/ journal.pone.0089692

39. Haberland NA. The case for addressing gender and power in sexuality and HIV education: a comprehensive review of evaluation studies. Int Perspect Sex Reprod Health. 2015;41(1):31-42. doi:10.1363/4103115

40. DiCenso A, Guyatt G, Willan A, Griffith L. Interventions to reduce unintended pregnancies among adolescents: systematic review of randomised controlled trials. BMJ. 2002;324(7351):1426. doi:10.1136/bmj.324.7351.1426

41. Carr JB, Packham A. The Effects of State-Mandated Abstinence-Based Sex Education on Teen Health Outcomes. Health Econ. 2017;26(4):403-420. doi:10.1002/hec.3315

42. Breunig M. Abstinence-Only Sex Education Fails African American Youth. J Christ Nurs. 2017;34(3):E41-e48. doi:10.1097/cnj.0000000000000409

43. Sharifi F, Sharifi N. The effect of educational intervention on lifestyle modification associated with osteoporosis in female students. The Iranian Journal of Obstetrics, Gynecology and Infertility. 2017;20(7):36-43. doi:10.22038/ ijogi.2017.9506

44. Saito MI. Sex education in school: preventing unwanted pregnancy in adolescents. Int J Gynaecol Obstet. 1998;63 Suppl 1:S157-160. doi:10.1016/S0020-7292(98)00199-4

45. Mosavi SA, Babazadeh R, Najmabadi KM, Shariati M. Assessing Iranian adolescent girls' needs for sexual and reproductive health information. J Adolesc Health. 2014;55(1):107-113. doi:10.1016/j.jadohealth.2013.11.029

46. Vivancos R, Abubakar I, Phillips-Howard P, Hunter PR. School-based sex education is associated with reduced risky sexual behaviour and sexually transmitted infections in young adults. Public Health. 2013;127(1):53-57. doi:10.1016/j.puhe.2012.09.016

47. Williams MT, Bonner L. Sex education attitudes and outcomes among North American women. Adolescence. 2006;41(161):1-14.

(C) 2019 The Author (s); This is an open-access article distributed under the terms of the Creative Commons Attribution License (http://creativecommons.org/licenses/by/4.0), which permits unrestricted use, distribution, and reproduction in any medium, provided the original work is properly cited. 